\title{
Ultrasound imaging through multimode waveguides using time reversal virtual array method
}

\author{
Mantvydas Kalibatas ${ }^{1}$, Richard Nauber ${ }^{1}$, David Dawidowski ${ }^{1}$, Lars Büttner ${ }^{1}$, Jürgen Czarske ${ }^{1}$ \\ ${ }^{1}$ Laboratory of Measurement and Sensor System Technique, \\ Faculty of Electrical and Computer Engineering, \\ Technische Universität Dresden, 01062 Dresden, Germany \\ mantvydas.kalibatas@tu-dresden.de
}

\begin{abstract}
:
In industrial processes, such as crystal growth of photovoltaic silicon or continuous steel casting, the quality of the end-products strongly depends on the melt flow. Therefore, in situ flow imaging of opaque melts under harsh conditions is important. However, ultrasound transducer arrays usually can not be operated at temperatures beyond $100^{\circ} \mathrm{C}$. An approach is to a use multimode waveguide, to spatially seperate the sensor from the measurement fluid. To overcome the complex ultrasound propagation through a multimode waveguide and enable imaging of the measurement volume, the time reversal virtual array (TRVA) method is used. Time invariance of wave equation in a linear medium allows refocusing on a limited set of calibrated points, which form the TRVA. In this paper, the TRVA has been characterized theoretically, numerically and experimentally. It has been shown that the virtual array can focus the ultrasound beam in transmit and receive modes. The achieved spatial resolution varies from $2 \mathrm{~mm}$ to $4 \mathrm{~mm}$ in experiments with water and $3 \mathrm{MHz}$ central transmit frequency. A planar velocity measurement of rotating flow in gallium-indium-tin is demonstrated through a $68 \mathrm{~mm}$ borosilicate waveguide. A comparison with a reference measurement showed a typical relative deviation smaller than $5,3 \%$.
\end{abstract}

Key words: ultrasound, imaging, time reversal, ultrasound multimode waveguide, virtual array.

\section{Introduction}

In industrial processes, such as photovoltaic silicon manufacturing or continuous steel casting, the quality, yield and energy efficiency can be improved by flow control. A promising approach is to affect the flow of electrically conductive fluids by using spatiotemporally varying magnetic fields, thus applying magnetohydrodynamics (MHD). The influence of the flow on material and heat transfer is investigated by means of numerical simulation and laboratory-scale model-experiments [1-3]. In low temperature models the phased array ultrasound Doppler velocimeter (PAUDV) is capable of providing planar velocity measurements with a spatial resolution of under $1 \mathrm{~mm}$ [4]. To create a closed loop control, the in-situ flow imaging of hot, opaque melts with temperatures beyond $1200^{\circ} \mathrm{C}$ is desirable. However, due to the Curie temperature of the ultrasound (US) sensors (PZT5A: $360^{\circ} \mathrm{C}$ [5]) a direct application to industrial processes is not possible. An approach to use ultrasound transducers in harsh environments is to separate them from the measurement volume through a waveguide. Previous investigations mostly employed mechanically complex single mode waveguides, which hinder planar flow imaging [6]. We propose the use of a multimode waveguide. To compensate the complex ultrasound propagation through the waveguide, the time reversal (TR) method is used [7]. It is based on the reciprocity of wave equation, also called time reversal invariance, and allows spatiotemporal refocusing to the initial source without any knowledge about the linear, stationary propagation channel. However, planar imaging with TR requires a large calibration effort [8]. For each point of interest a physical beacon has to be present, to which TR procedure can be performed. This is impractical in most applications and a suggested solution is employing a time reversal virtual array (TRVA). Therefore only a limited set of precalibrated points are needed, which form the virtual array. The TRVA is a combination of the TR principle, to compensate the complex wave propagation in the waveguide, and of the conventional phased-array technique, to employ the imaging beyond the virtual array. The conventional signal processing strategies for phased-arrays, like transmit (TX) and receive (RX) beamforming [9] and ultrasound Doppler 
velocimetry (UDV) [10], can be applied to TRVA.

\section{Concept of TRVA}

Robert and Fink were the first, who presented the idea of a TR based virtual array [11]. Walker et. al. developed this idea further and named the concept "Virtual Source Array" (VSA) for the application in sonar acoustics [12]. We extend this concept to combine TX and RX beamforming and call it TRVA.

TRVA combines the TR method with the conventional phased-array technique. The TRVA is remotely operated by a physically present US-Array, called time reversal mirror (TRM), and can be conceptually treated as a phased-array. This enables an application of active coherent focusing techniques and even velocity estimation algorithms for imaging beyond the virtual array through the waveguide.

Multiple, linearly placed US point sources, which are also called virtual transducers, define the TRVA. The US point source can be approximated by a focused US beam at the focus location [11]. The focusing is realized by the TR principle. At a calibration step, an element of TRVA initiates an ultrasound pulse, which propagates through a complex medium and is recorded by the TRM. The received, windowed and time reversed field is a so called TR pattern $P_{i}$ of the considered virtual transducer $i$. If this TR pattern is retransmitted by the TRM, waves of the time reversed ultrasound field retraces all the complex propagation paths and interfere constructively at the location of the virtual source. To capture the full transfer matrix between TRM and TRVA each element of TRVA has to be calibrated.

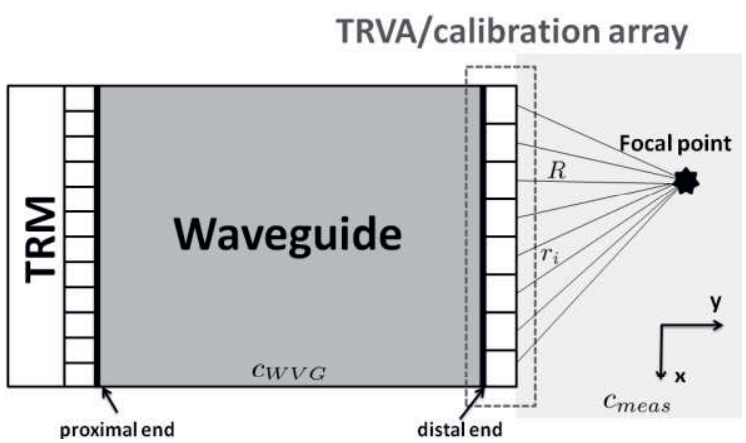

Fig. 1. Focusing with a TRVA: the calibration of the TRVA compensates the complex propagation from the TRM at the proximal end to the distal end. Conventional beamforming is used to achieve focusing at a focal point.

The calibration is realized by attaching a physically present linear transducer array at the distal end of the waveguide. This array is called calibration array and its geometry corresponds to that of the TRVA (see fig. 1.).
TRVA can be conceptually treated as a conventional phased-array and therefore beamforming algorithms can be applied. For TX focusing to a point beyond the virtual array, phased-delayed TR patterns has to be summed for each transmitting element of TRM. The delays $\tau_{i}$ are calculated corresponding to standard time-of-flight equation (see eq. 1.), whereby $r_{i}$ is the distance between respective virtual transducer and focal point, $R$ the shortest distance and $c_{\text {meas }}$ the sound speed in measurement medium (see Fig. 1.).

$\tau_{i}=\frac{r_{i}-R}{c_{\text {meas }}}$

Received signals of the TRVA $r^{V A}$ are extracted by executing the convolution between the echo signals recorded by the TRM $r^{R A}$ and the TR patterns, like in equation (2). With the present receive signals of TRVA $r^{V A}, \mathrm{RX}$ focusing can be applied [9].

$r_{i}^{V A}(t)=\left(r_{i}^{R A} * P_{i}\right)(t)$

Directivity of the TRVA is limited. Time reversed ultrasound field, transmitted by the virtual transducer, approximates the original sound field of a beacon with a main difference, that it is not isotropic. The anisotropy limits the directivity pattern of the whole TRVA and prevents large beam steering angles, which could be achieved with a real phased-array. This limitation is dependent on the critical angle of the waveguide-fluid interface [12].

\section{Numerical characterization}

In order to characterize the imaging capabilities of a TRVA-system, numerical simulations using the elastodynamic finite integration technique (EFIT) to model two-dimensional wave propagation in elastic media are conducted [14]. Criteria for evaluating the resulting sound fields in case of TX beamforming and point spread functions (PSF) in case of $R X$ beamforming are the full width at half maximum (FWHM) and the focus-to-background ratio (FBR). FWHM describes the lateral resolution as an ability to separate two objects perpendicular to the propagation axis. FBR represents the relation between ultrasound field intensity at the focus to the mean intensity of the background.

Spatial distribution of FWHM and FBR has been simulated to characterize the focusing ability of the TRVA with the parameters in table 1. Thereby $f_{0}$ is center transmit frequency, $p$ pitch length, $N$ element count of the array, $Z$ specific acoustic impedance, $L_{W V G}$ and $W_{W V G}$ represent length and width of the waveguide. The waveguide and measurement medium are both water. The spatial distribution of evaluation 
criteria, which is extracted from sound field of TX focusing with the TRVA, can be found in fig. 2. The achieved spatial resolution varies from approx. 1 to $4 \mathrm{~mm}$. In fig. 3. the distribution of the criteria from PSF of RX focusing is shown. The FWHM is in range from 0.4 to $2.8 \mathrm{~mm}$ and FBR $>200$. With increasing distance from the array or bigger steering angles the ability to focus decreases.

Tab. 1: Configuration of numerical simulations

\begin{tabular}{|c|c||c|c|}
\hline$f_{0_{T R M}}$ & $3 \mathrm{MHz}$ & $f_{0_{\text {calib }}}$ & $3 \mathrm{MHz}$ \\
\hline$p_{T R M}$ & $\begin{array}{c}0.2495 \\
\mathrm{~mm}\end{array}$ & $p_{V A}$ & $\begin{array}{c}0.2495 \\
\mathrm{~mm}\end{array}$ \\
\hline$N_{T R M}$ & 32 & $N_{V A}$ & 32 \\
\hline$c_{W V G}$ & $1497 \mathrm{~m} / \mathrm{s}$ & $c_{\text {meas }}$ & $1497 \mathrm{~m} / \mathrm{s}$ \\
\hline$Z_{W V G}$ & $\begin{array}{c}1.5 \\
\mathrm{MPa} \cdot \mathrm{s} / \mathrm{m}\end{array}$ & $Z_{\text {meas }}$ & $\begin{array}{c}1.5 \\
\mathrm{MPa} \cdot \mathrm{s} / \mathrm{m}\end{array}$ \\
\hline$L_{W V G}$ & $136 \mathrm{~mm}$ & $W_{W V G}$ & $34 \mathrm{~mm}$ \\
\hline
\end{tabular}
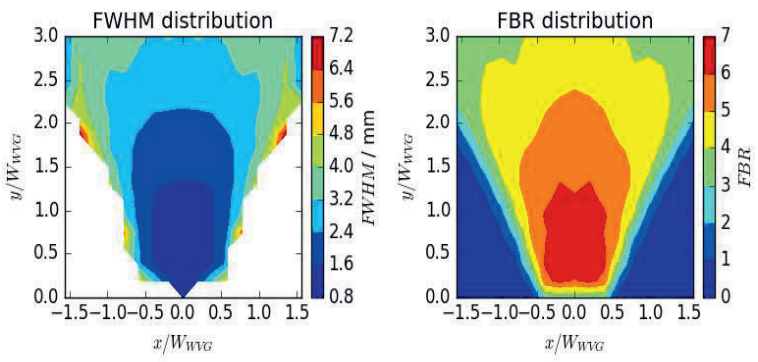

Fig. 2. Simulated spatial distribution of FWHM and FBR from sound field data, which is acquired by $T X$ focusing with TRVA.
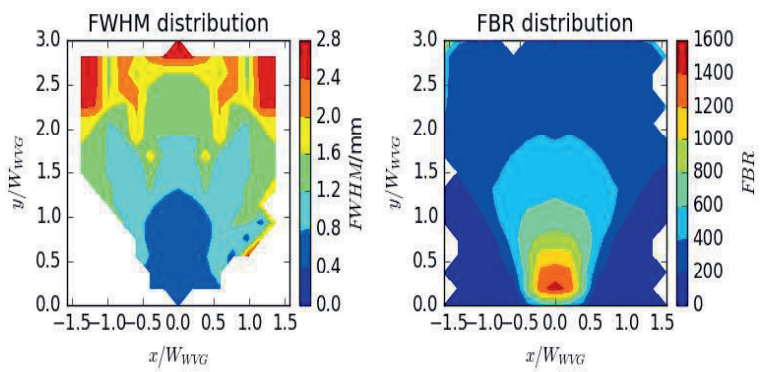

Fig. 3. Simulated spatial distribution of FWHM and FBR from point spread functions, which are acquired by $R X$ focusing with TRVA.

\section{Experimental characterization}

The application of TRVA is characterized in a reference experiment to prove the functionality of the concept. The quality of TR calibration is analyzed to demonstrate the refocusing behavior by the TRM through the waveguide. Also, the ability to focus the beam in TX and RX modes, as well as estimate the velocity with the TRVA is investigated. The configuration of the experiment is listed in table 2 , whereby $P R F$ is the pulse repetition frequency and $U_{T R M}$ the excitation voltage of the TRM. The PAUDV system [4] is used to operate the TRM. The waveguide consists of borosilicate glass and the measurement volume is water.

Tab. 2: Configuration of model experiments in water

\begin{tabular}{|c|c||c|c|}
\hline$f_{0_{T R M}}$ & $3 \mathrm{MHz}$ & $f_{0_{\text {calib }}}$ & $4 \mathrm{MHz}$ \\
\hline$p_{T R M}$ & $0.5 \mathrm{~mm}$ & $p_{V A}$ & $0.3 \mathrm{~mm}$ \\
\hline$N_{T R M}$ & 64 & $N_{V A}$ & 96 \\
\hline$c_{W V G}$ & $6050 \mathrm{~m} / \mathrm{s}$ & $c_{\text {meas }}$ & $1497 \mathrm{~m} / \mathrm{s}$ \\
\hline$Z_{W V G}$ & $\begin{array}{c}13.5 \\
\mathrm{MPa} \cdot \mathrm{s} / \mathrm{m}\end{array}$ & $Z_{\text {meas }}$ & $\begin{array}{c}1.5 \\
\mathrm{MPa} \cdot \mathrm{s} / \mathrm{m}\end{array}$ \\
\hline$L_{W V G}$ & $136 \mathrm{~mm}$ & $W_{W V G}$ & $34 \mathrm{~mm}$ \\
\hline$P R F$ & $50 \mathrm{~Hz}$ & $U_{T R M}$ & $20 \mathrm{~V}$ \\
\hline
\end{tabular}

The quality of TRVA calibration has been examined by refocusing to each virtual transducer. A cross-section of the sound field measured by calibration array is shown in fig. 4., when refocusing to element 26 . of the TRVA. The mean FBR of all virtual transducers is 6.91 and the mean FWHM is $1.96 \mathrm{~mm}$, which corresponds to $0.97 \lambda_{\text {boro }}$ (wavelength in borosilicate).

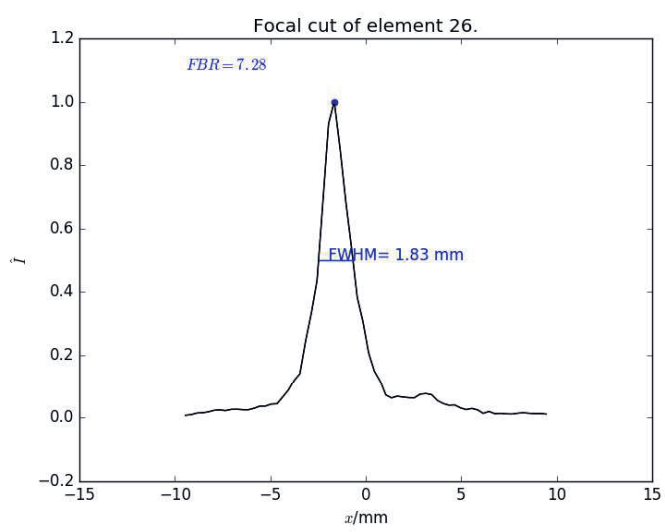

Fig. 4. Focus cut of experimentally refocusing to element 26. of TRVA at expected time. Blue point corresponds to expected focus peak.

The sound field scanned with a hydrophone (diameter $d=1 \mathrm{~mm}$ ) of a single TX focus point located at $\left(0, W_{W V G}\right)$ is illustrated in fig. 5. left. The focus is well resolved with a lateral resolution of $3.09 \mathrm{~mm}$, FBR of 22.2 and there are no visible side lobes. In figure 6 . a plane wave transmitted by the TRVA is demonstrated. Intensity curve approximates the expected plane wave but shows a decline at the waveguide edges. This apodisation effect is due to the increased attenuation of longer propagation paths in the waveguide [15]. 

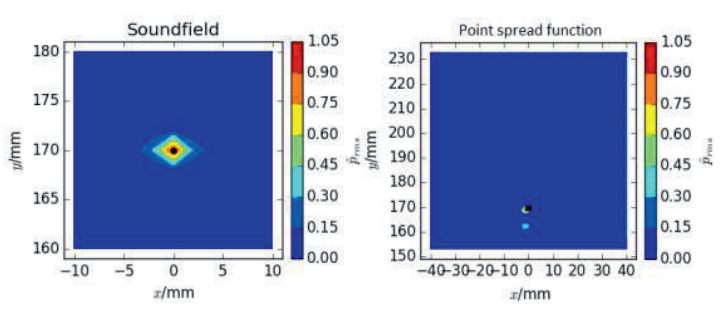

Fig. 5. Experimentally measured sound field of $T X$ focusing (left) and point spread function of $R X$ focusing (right) to the position $(0,170 \mathrm{~mm})$ with center of the virtual array at $(0,136 \mathrm{~mm})$. Black point corresponds to expected focus location.

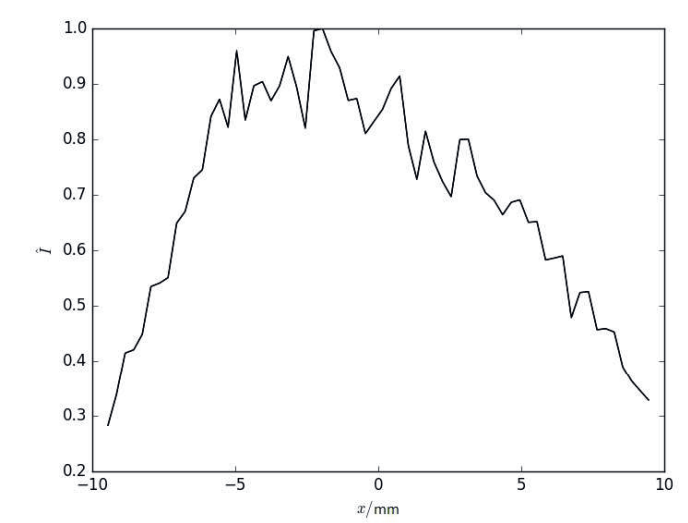

Fig. 6. Experimentally measured sound field at position of TRVA, when transmitting a plane wave.

In fig. 5. right $R X$ focusing to the same point as for TX focusing (fig. 5. left) is illustrated. The FWHM of PSF is $2 \mathrm{~mm}$ and the FBR is 364 .
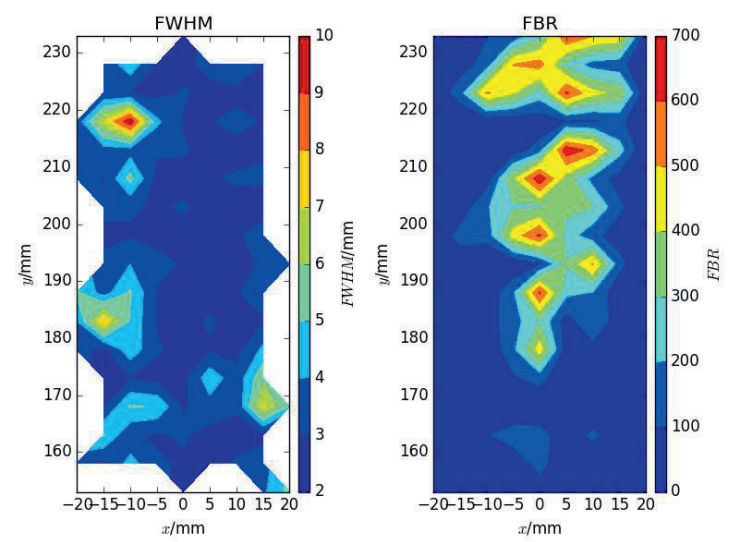

Fig. 7. Spatial distribution of evaluation criteria for experimental RX focusing with the TRVA. Center of TRVA at $(0,136 \mathrm{~mm})$.

An experimentally obtained map of spatial FWHMs and FBRs distribution for RX beamforming is presented in fig. 7. The FWHM varies between 2 and $4 \mathrm{~mm}$ for most of the observed regions. This shows an agreement to the simulation results within an absolute difference of about $1 \mathrm{~mm}$ (compare with fig. 3). However, a strongly limited spatial directivity cone of TRVA can be recognized from FBR map, because of the critical angle of the waterborosilicate interface $\left(\theta_{\text {crit }}=14.3^{\circ}\right)$.

Lastly, the ability to measure velocities through UDV [10] with the TRVA was tested. TRVA was periodically transmitting plane waves, while a scatterer with diameter of $0.1 \mathrm{~mm}$ was moving away from the virtual array with reference velocity of $3.125 \mathrm{~mm} / \mathrm{s}$. Over 20 measurements estimated mean velocity value is $3.121 \mathrm{~mm} / \mathrm{s}$ with standard deviation of $0.025 \mathrm{~mm} / \mathrm{s}$. This results in a relative error of $0.128 \%$.

\section{Experimental flow imaging with TRVA}

An experiment with the TRVA in a cube with liquid metal was performed to image the flow and evaluate the uncertainty compared to a reference measurement. In a low temperature alloy of gallium-indium-tin (GalnSn) rotating flow was induced by a spatiotemporally varying magnetic field. The TRVA is positioned on the distal end of the waveguide, which is attached to the wall of the cube (6 mm PMMA). The experimental configuration is listed in table 3 . and the setup is illustrated in figure 8 .

Tab. 2: Configuration of model experiments in GalnSn cube.

\begin{tabular}{|c|c||c|c|}
\hline$f_{0_{T R M}}$ & $3 \mathrm{MHz}$ & $f_{0_{\text {calib }}}$ & $4 \mathrm{MHz}$ \\
\hline$p_{T R M}$ & $0.5 \mathrm{~mm}$ & $p_{V A}$ & $0.3 \mathrm{~mm}$ \\
\hline$N_{T R M}$ & 64 & $N_{V A}$ & 96 \\
\hline$c_{W V G}$ & $6050 \mathrm{~m} / \mathrm{s}$ & $c_{\text {meas }}$ & $2730 \mathrm{~m} / \mathrm{s}$ \\
\hline$Z_{W V G}$ & $\begin{array}{c}13.5 \\
\mathrm{MPa} \cdot \mathrm{s} / \mathrm{m}\end{array}$ & $Z_{\text {meas }}$ & $\begin{array}{c}17.4 \\
\mathrm{MPa} \cdot \mathrm{s} / \mathrm{m}\end{array}$ \\
\hline$L_{W V G}$ & $68 \mathrm{~mm}$ & $W_{W V G}$ & $34 \mathrm{~mm}$ \\
\hline$P R F$ & $200 \mathrm{~Hz}$ & $U_{R e f \mid} U_{T R M}$ & $12 \mathrm{~V} / 18 \mathrm{~V}$ \\
\hline
\end{tabular}

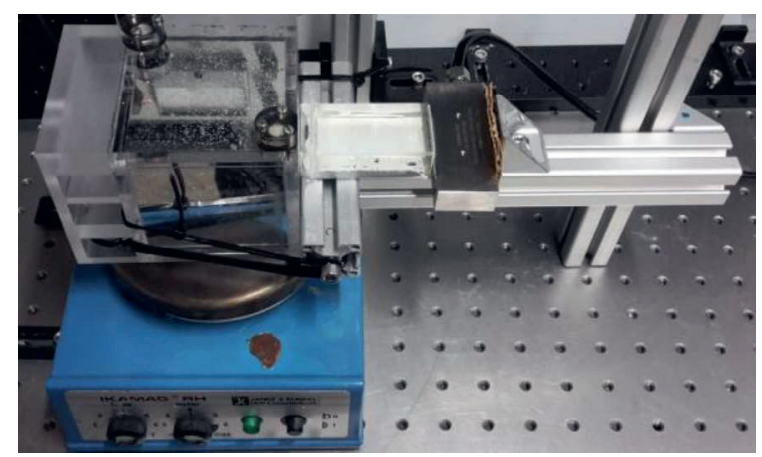

Fig. 8. Experimental setup of flow imaging in a Galnsn cube with TRVA through borosilicate waveguide. 

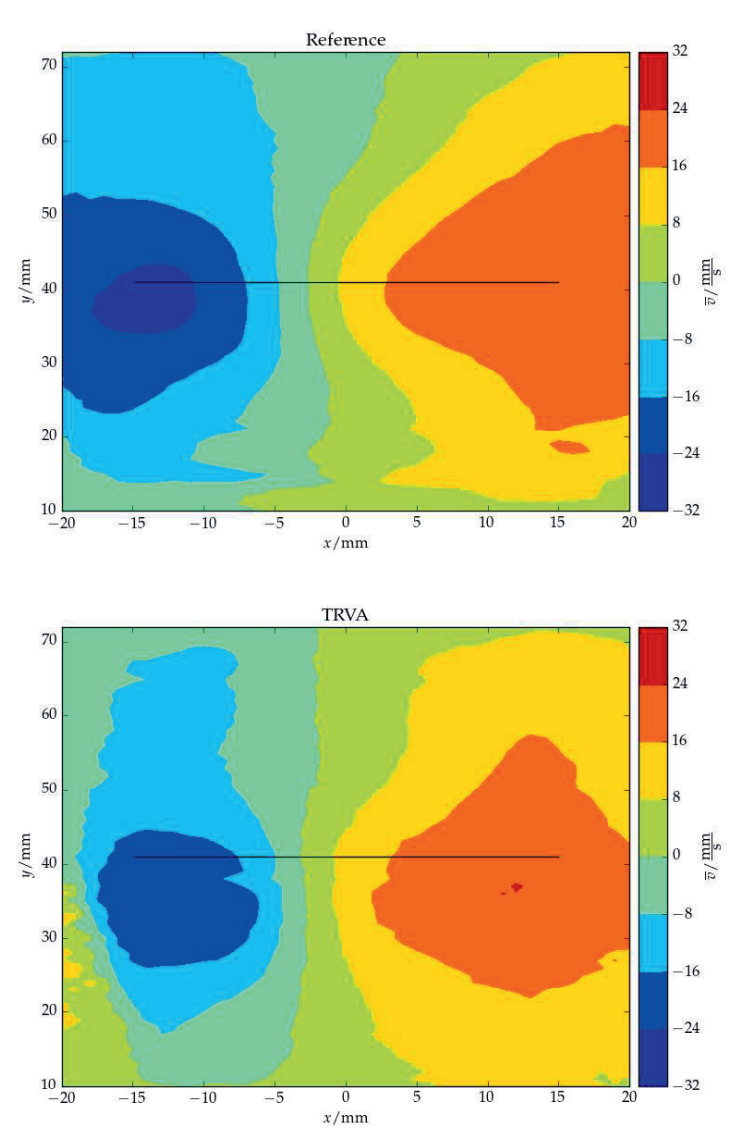

Fig. 9. Experimental imaging (2d-1c) of counterclockwise rotating mean flow induced in GalnSn cube over 250 frames, which was measured by the phased-array directly placed on the cube (top) and by the TRVA through a borosilicate waveguide (bottom). Black line indicates the position of the velocity profile in figure 10.

The induced velocity field of the fluid in the cube is unknown, therefore a reference flow measurement without a waveguide is performed. The counterclockwise rotating flow is measured in a plane with one velocity component (2d-1c).

In fig. 9. the reference flow measurement is compared to the measurement with the TRVA for the average of 250 frames. The qualitative flow behavior measured with the TRVA is similar to the reference. However, also here limited directivity causes velocity artifacts outside of the lateral range of the TRVA $x \in[-14,4 ; 14,4] \mathrm{mm}$.

A quantitative comparison for a velocity profile at $y=41 \mathrm{~mm}$ is given in figure 10 . The mean velocity differs only by maximal $5,3 \%$ for $x \in[0$; 15] $\mathrm{mm}$. For $x \in[-15 ;-5] \mathrm{mm}$ a relative deviation rises to $25 \%$. This could be the result of increased spatial averaging of the TRVA measurement. However, these results demonstrate, that the TRVA can be used to compensate the complex propagation in the waveguide and to do the imaging as with the conventional phased-array.

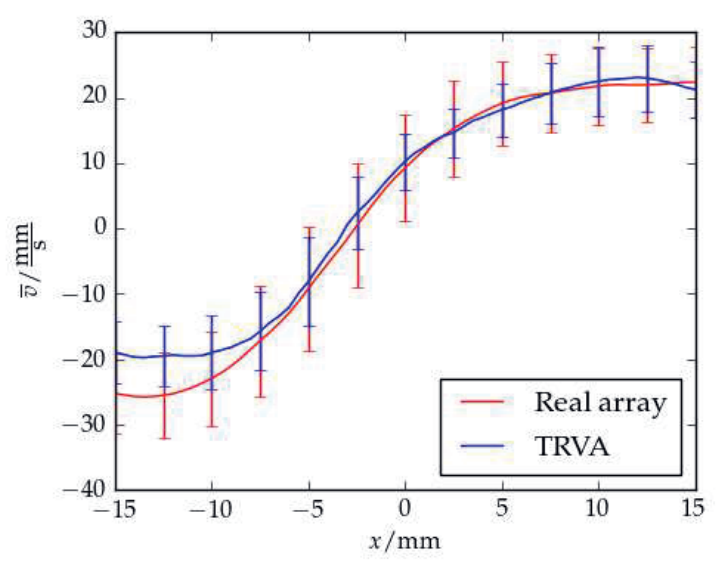

Fig. 10. Experimentally estimated mean velocity at averaged depth $y=41 \mathrm{~mm}$ for the reference measurement and by the TRVA. Vertical bars correspond to the standard deviation.

\section{Conclusion}

TRVA is a concept, which combines the TR method with the phased-array technique to allow imaging through a multimode waveguide with reduced calibration effort. It allows to remotely operate virtual array by the real TRM. To compensate the unknown, complex wave propagation in the waveguide between the real and the virtual arrays, the calibration of only limited set of beacons, which define the TRVA, is needed. Because the virtual array can be conceptually considered as a conventional phased-array, any kind of signal processing, like TX or RX beamforming and UDV, could be applied to it.

In this paper, the TRVA was characterized theoretically, numerically and experimentally. A focusing on transmit and receive was demonstrated through a waveguide. The achieved lateral resolution in water with borosilicate waveguide of length $136 \mathrm{~mm}$ varies between 2 and $4 \mathrm{~mm}$ in a spatial TRVA directivity cone, which is limited by the critical angle. In model experiments with GalnSn induced flow was imaged with the TRVA through a waveguide with an estimated velocity uncertainty below $25 \%$.

The concept of TRVA may allow to image flows of hot melts in industrial processes, such as photovoltaic silicon manufacturing or continuous steel casting. Future investigation should target the robustness of the TRVA calibration regarding temperature gradients and deformations. 


\section{Acknowledgements}

We like to thank D. Räbiger and S. Eckert from Helmholtz-Zentrum Dresden-Rossendorf for providing the cube with GalnSn.

\section{References}

[1] L. Büttner, R. Nauber, M. Burger, D. Räbiger, S. Franke, S. Eckert, J. Czarske, Dual-plane ultrasound flow measurements in liquid metals. Measurement Science and Technology 24(5), (2013); doi: 10.1088/0957-0233/24/5/055302

[2] R. Nauber, M. Burger, M. Neumann, L. Büttner, K. Dadzis, K. Niemietz, O. Pätzold, J. Czarske, (2013). Dual-plane flow mapping in a liquid-metal model experiment with a square melt in a traveling magnetic field, Experiments in fluids 54(1502), (2013); doi: 10.1007/s00348-013-1502$\mathrm{x}$

[3] R. Nauber, N. Thieme, H. Radner, H. Beyer, L. Büttner, K. Dadzis, O. Pätzold, J. Czarske, Ultrasound flow mapping of complex liquid metal flows with spatial self-calibration, Flow Measurement and Instrumentation 48, 59-63 (2016); doi: 0.1016/j.flowmeasinst.2015.12.005

[4] K. Mäder, R. Nauber, H. Beyer, A. Klaß, C. Kupsch, N. Thieme, L. Büttner, J. Czarske, J, Adaptive Ultrasound Doppler Velocimeter for the investigation of turbulent flows in liquid metals, 18. GMA/ITG-Fachtagung Sensoren und Messsysteme (2016); doi: 10.5162/sensoren2016/5.4.3

[5] H. J. Lee, S. Zhang, Y. Bar-Cohen, S. T. Sherrit, High Temperature, High Power Piezoelectric Composite Transducers, Sensors(Basel) 14(8), 14526-14552 (2014); doi: 10.3390/s140814526

[6] S. Eckert, S. Franke, T. Gundrum, G. Gerbeth, J.C. Willemetz, Applications of Ultrasonic Doppler Velocimetry to flow measurements in hot liquid metals, 8th International Conference on Electromagnetic Processing of Materials, (2015)

[7] M. Fink, Time Reversal of Ultrasonic Fields - Part I: Basic Principles, IEEE Transactions on Ultrasonics, Ferroelectrics, and Frequency Control 39(5), 555-566 (1992); doi: 10.1109/58.156174

[8] D. Dawidowski, R. Nauber, L. Büttner, J. Czarske, Time reversal ultrasound focusing through multimode waveguides, tm-Technisches Messen, (2017); doi: 10.1515/teme-2016-0062

[9] O. T. Ramm, F. L. Thurstone, Cardiac Imaging Using a Phased Array Ultrasound System, Circulation 53(2), 258-262 (1976); doi: 10.1161/01.CIR.53.2.258

[10] T. Loupas, R. W. Gill, An Axial Velocity Estimator for Ultrasound Blood Flow Imaging, Based on a Full Evaluation of the Doppler Equation by Means of a Two-Dimensional Autocorrelation Approach, IEEE Transactions on Ultrasonics, Ferroelectrics, and Frequency Control 42(4), 672 - 688 (1995); doi: $10.1109 / 58.393110$
[11] J. L. Robert, M. Fink, The time-reversal operator with virtual transducers: Application to far-field aberration correction, Acoustical Society of America 126(6), 3659-3668 (2008); doi: 10.1121/1.3005560.

[12] S.C. Walker, P. Roux, W.A. Kuperman, Synchronized time-reversal focusing with application to remote imaging from a distant virtual source array, Acoustical Society of America 125(6), 3828-3834 (2009); doi: 10.1121/1.3117374

[13] W. R. Hedrick, D. L. Hykes, D. E. Starchman. Ultrasound Physics and Instrumentation 4, (2005) isbn: 978-0-323-03212-4

[14] M. Molero-Armenta, U. Iturrarán-Viveros, S. Aparicio, M. G. Hernández, Optimized OpenCL implementation of the Elastodynamic Finite Integration Technique for viscoelastic media, Computer Physics Communications 185(10), 2683-2696 (2014), doi: 10.1016/j.cpc.2014.05.016

[15] P. Roux, B. Roman, M. Fink, Time-reversal in an ultrasonic waveguide, Applied Physics Letters 70(14), 1811-1813 (1997), doi: 10.1063/1.118730 